\title{
CUANTIFICACIÓN DE LA MASA MUSCULAR DE LOS MIEMBROS APENDICULARES, POR MEDIO ECUACIONES ANTROPOMÉTRICAS
}

\author{
MUSCLE MASS QUANTIFICATION OF APPENDICULAR MEMBERS, TROUGH \\ ANTHROPOMETRIC EQUATIONS
}

\author{
Barraza Gómez, Fernando; Hadler Garcés, Adolfo; Jeria Fernández, José \& Riffo Esquivel, Carolina. \\ Laboratorio de Motricidad Humana, Escuela de Educación Física, Pontificia Universidad Católica de Valparaíso, \\ Chile
}

\begin{abstract}
BARRAZA, G.F.; HADLER, G.A.; JERIA, F.J.; RIFFO, E.C. Cuantificación de la masa muscular de los miembros apendiculares, por medio ecuaciones antropométricas. Rev. Motr. Hum., 10(2): 44-48, 2009.

\section{RESUMEN}

En 1990, Alan Martin publica una ecuación de regresión para la estimación de la masa muscular y la correlaciona con disecciones de 12 cadáveres, obteniendo un coeficiente de determinación $\left(R^{2}\right)$ de 0,97 (o sea, excelente para predecir la masa de los cadáveres) y el error de estimación estándar (EEE) 1,53 kilos (kg). En 1997, un canadiense de nombre Malcolm Doupe modifica la ecuación de Martin para calcular la masa muscular de los 23.400 canadienses que fueron medidos en el Canada Fitness Survey de 1981, donde no se midió ni el perímetro de muslo medio ni el de antebrazo que requieren la ecuación de Martin, pero sí los perímetros de muslo máximo y brazo relajado. Doupe presenta la siguiente ecuación: Masa muscular $(\mathrm{g})=$ talla $(\mathrm{cm}) *\left(0,031 * M U T h G^{2}+0,064 * C C G^{2}+0,089 * C A G^{2}\right)-3,006$.

La fórmula para el cálculo de la masa muscular del miembro superior es M SUP=talla $(\mathrm{cm}) *\left(0,089 * C A G^{2}\right)-3,006$ y para el miembro inferior y abdomen, $M I N F=$ talla $(\mathrm{cm}) *\left(0,031 * M U T h G^{2}+0,064 * C C G^{2}\right)-3,006$.

Conociendo esta ventaja, se aplican las fórmulas a dos equipos de deportistas chilenos, 11 jugadores profesionales de rugby y 11 jugadores profesionales de futbol.

Se obtienen diferencias significativas para $p>0,05$ entre los $\mathrm{kg}$ de masa muscular total, donde, Rugby= 48,1 kg; Fútbol= $42,3 \mathrm{~kg}$ y del miembro superior, siendo Rugby= 16,1 kg; Fútbol= 12,5kg. En los miembros inferiores hay diferencias significativas de p > 0,05 en los porcentajes (\%) de masa muscular, obteniendo Rugby=35\%; Fútbol= 40,9\%.

Por medio de este método es posible precisar no sólo el componente total de masa muscular, sino que identificar la ubicación en mayor o menor presencia en miembros superiores, abdomen o miembro inferior, permitiendo comparar de forma más precisa la distribución de la masa muscular de distintos sujetos.
\end{abstract}

Palabras Clave: Antropometría, Composición Corporal, Deporte.

\section{INTRODUCCIÓN}

La antropometría se ha convertido en el método más práctico para la determinación de los componentes anatómicos del cuerpo humano (1). En un comienzo, sólo definiendo la cantidad de grasa corporal y masa muscular, contribuyendo autores como Andreas Vesalius y Adolf Quetelet en los siglos XVI y XIX respectivamente, dando paso a los trabajos realizados a comienzos del siglo $\mathrm{XX}$ y desarrollando fórmulas regresión múltiple para estimación de los componentes corporales a partir de la densidad corporal $(2,3,4)$. Jindrich Matiegka, considerado el padre de la Antropometría, crea el primer método para la cuantificación de la composición corporal en 4 componentes: masa muscular, masa grasa, masa ósea y masa residual (5). Estos aportes han contribuido enormemente en la mejora de los métodos de composición corporal, que dan una idea precisa de la cantidad presente de cada componente morfoestructural. Uno de los componentes que define el funcionamiento físico humano, es la masa muscular, cual está relacionada con variables biomecánicas como la fuerza y la velocidad (6).

En su tesis doctoral, Donald Drinkwater plantea la idea de seccionar los componentes corporales por medio de ecuaciones regresivas que permiten obtener dichos componentes, 
reconociendo a las extremidades y tronco como cilindros y conos, obteniendo una idea de cada componente por región anatómica $(7,8)$, concepto que varios autores rechazan.

En 1990, Alan Martin publica una ecuación de regresión para la estimación de la masa muscular y la correlaciona con disecciones de 12 cadáveres (9), obteniendo un coeficiente de determinación $\left(\mathrm{R}^{2}\right)$ de 0,97 (o sea, excelente para predecir la masa de los cadáveres) y el error de estimación estándar (EEE) 1,53 kg (también muy bueno). Pero como es una ecuación de regresión múltiple, tiene la particularidad de ser muy representativa de la muestra, que en este caso eran ancianos belgas masculinos, por lo tanto no debería utilizarse esta ecuación en otros grupos. En 1997, un canadiense de nombre Malcolm Doupe modifica la ecuación de Martin para calcular la masa muscular de los 23.400 canadienses que fueron medidos en el Canada Fitness Survey de 1981, donde no se midió ni el perímetro de muslo medio ni el de antebrazo que requieren la ecuación de Martin, pero sí los perímetros de muslo máximo y brazo relajado (10).

A partir de esta fórmula es posible obtener resultados de la cantidad de masa muscular dividida en dos grandes componentes, "miembro superior" y "tronco con miembro inferior"; lo que permite tener una idea más precisa de la distribución de la masa muscular en determinados sujetos y no sólo de manera general como se hace hasta ahora.

\section{SUJETOS Y MÉTODOS}

Utilizamos como referencia la fórmula para determinar el componente muscular de los individuos de Malcolm Doupe del año 1997. Observamos que hay tres elementos anatómicos de importancia que componen esta fórmula, medidas del miembro superior que son el perímetro del brazo relajado y el pliegue del tríceps y medidas del miembro inferior que son el perímetro del muslo medio, el perímetro de la pierna, el pliegue de la pierna y el pliegue supraespinal que comprende anatómicamente a la zona abdominal lateral.

Masa muscular $(\mathrm{g})=$ talla $(\mathrm{cm}) *$ $\left(0,031 *\right.$ MUThG $^{2}+0,064 * \mathrm{CCG}^{2}+$ $\mathbf{0 , 0 8 9} * \mathbf{C A G}^{2}$ ) - 3,006 donde, MUThG = perímetro de muslo máximo corregido por pliegue supra-espinal.

CCG = perímetro de pantorrilla máximo corregido por pliegue homónimo.

$\mathrm{CAG}=$ perímetro de brazo relajado corregido por pliegue de tríceps.

Perímetro corregido $=$ Perímetro del miembro (en $\mathrm{cm})$ - (pliegue del miembro (en $\mathrm{cm})^{*}$ pi).

Tomamos las variables antropométricas del miembro superior y aplicamos la fórmula con las mismas constantes, lo mismo para las variables que representan la masa muscular del miembro inferior.

La fórmula para el cálculo de la masa muscular del miembro superior (M SUP) estaría representada como:

$$
\text { M SUP }=\text { talla }(\mathrm{cm}) *\left(0,089 * \mathrm{CAG}^{2}\right)-3,006
$$

La fórmula para el cálculo del miembro inferior y tronco (M INF) estaría representada como:

M INF $=$ talla $(\mathrm{cm}) *\left(0,031 * \mathrm{MUThG}^{2}+\right.$ $\left.0,064 * C^{2} G^{2}\right)-3,006$.

La suma de las ambas fórmulas tiene como resultado la masa muscular obtenida con la fórmula original. Comprobamos y validamos el procedimiento obteniendo los resultados adecuados.

Conociendo esta simple modificación, se aplican las fórmulas a dos equipos de deportistas, conformados por 11 jugadores profesionales de rugby de Chile y 11 jugadores profesionales de fútbol de Chile (Tabla 1). 


\begin{tabular}{lcccc} 
& \multicolumn{2}{c}{ RUGBY } & \multicolumn{2}{c}{ FÚTBOL } \\
& Media & D.E. & Media & D.E. \\
\hline Peso & 93,3 & 15,6 & 78,4 & 25,2 \\
Estatura & 179,6 & 6,8 & 172,6 & 18,4 \\
Pliegue Tríceps & 8,5 & 5,6 & 5,3 & 2,5 \\
Pliegue Subescapular & 12,7 & 8,4 & 7,3 & 4,1 \\
Pliegue Supracrestideo & 15,5 & 14,2 & 3,0 & 4,1 \\
Pliegue Abdominal & 17,9 & 10,3 & 14,3 & 9,9 \\
Pliegue Muslo & 13,4 & 9,8 & 5,0 & 2,3 \\
Pliegue Pierna & 7,8 & 4,6 & 2,0 & 1,3 \\
Perímetro del Brazo & 34,2 & 3,2 & 30,6 & 2,3 \\
Perímetro del Muslo & 58,8 & 5,3 & 53,0 & 4,1 \\
Perímetro de la Pierna & 39,4 & 4,1 & 38,4 & 2,3
\end{tabular}

Tabla 1: Características antropométricas de ambos grupos de sujetos utilizados para comprobar la ecuación. D.E.= Desviación Estándar.

Para la recolección de las medidas y para el protocolo de medición se usó un kit antropométrico Rosscraft SRL, validado por la Internacional Society for the Avancement in Kineanthropometric (ISAK), que incluye antropómetros óseos, huincha métrica y plicómetro o calibre para pliegues. Las mediciones se realizaron bajo el protocolo de marcaje y medición de la ISAK, en condiciones normales de temperatura, a primera hora de la mañana y después del vaciado urinario, por evaluadores con licencia nivel II de dicha sociedad.

Los datos se registraron en planillas de recolección manual $\mathrm{y}$, posteriormente, en planillas Excel de Microsoft Office 2007 para su análisis estadístico básico.

\section{RESULTADOS}

Al utilizar esta adaptación de la fórmula para la predicción de la masa muscular, se obtienen diferencias significativas de $p<0,05$ en una prueba de "t", entre los kilos ( $\mathrm{kg}$ ) de masa muscular total entre ambos grupos, donde los kilos para el grupo de Rugby fue de $48,1 \mathrm{~kg}$ y para el grupo de Fútbol 42,3 kg.

En el miembro superior la cantidad de músculo del grupo de Rugby es de $16,1 \mathrm{~kg}$ y en el grupo de Fútbol fue $12,5 \mathrm{~kg}$, diferencias estadísticamente significativas obteniendo $\mathrm{p}<$ 0,05 en la prueba " $t$ ".

La cantidad de masa muscular que representan los miembros inferiores y tronco, muestra diferencias significativas de $\mathrm{p}<0,05$ en los porcentajes $(\%)$ de masa muscular, obteniendo un $35 \%$ de masa muscular para el grupo de Rugby y un $40,9 \%$ de masa muscular en el grupo de Fútbol. 


\begin{tabular}{llcc} 
& & RUGBY & FÚTBOL \\
\hline \multirow{3}{*}{ MÚSCULO (KG) } & Total & 48,1 & $42,3\left(^{*}\right)$ \\
& Miembro Superior & 16,1 & $12,4\left(^{*}\right)$ \\
& Tronco y Miembro Inferior & 32,0 & 29,8 \\
\hline \multirow{3}{*}{ MÚSCULO (\%) } & Total & 52,6 & 57,7 \\
& Miembro Superior & 17,6 & 16,9 \\
& Tronco y Miembro Inferior & 35,0 & $40,8\left(^{*}\right)$ \\
\hline
\end{tabular}

Tabla 2: Diferencias en la distribución de la masa muscular en miembro superior, tronco y miembro inferior de los grupos de jugadores profesionales de Rugby y Fútbol. (*) Diferencias estadísticas de $\mathrm{p}<0,05$ con respecto al grupo de jugadores de Rugby.

\section{DISCUSIÓN}

En la Tabla 2 se aprecian las diferencias en la cantidad de masa muscular entre ambos grupos, siendo más alta en el grupo de Rugby. Estos tipos de comparaciones dan una idea de la cantidad total de masa muscular que permite comparar grupos de sujetos como otros estudios hechos en Chile $(11,12)$, pero no determina su distribución de dicho componente. Las diferencias encontradas entre estos grupos de deportistas permiten establecer que hay diferencias importantes en la distribución del componente muscular, siendo más alto en los miembros superiores en jugadores de Rugby, dado su uso donde prevalecen los movimientos de agarre y empuje del rival; a diferencia del grupo de Fútbol, que presenta un porcentaje de masa muscular del tronco y miembro inferior mayor, debido a que su juego depende exclusivamente de los movimientos del miembro inferior. Más importante que las diferencias encontradas, señalamos que a partir de esta modificación de la fórmula es posible obtener resultados de la cantidad de masa muscular dividida en dos grandes componentes, "miembro superior" y "tronco con miembro inferior", lo que permite tener una idea más precisa de la distribución de la masa muscular para comparar distintos sujetos y grupos de sujetos que realizan actividades físicas diversas, precisando de esta manera los componentes estructurales que definen el rendimiento deportivo.

\section{REFERENCIAS BIBLIOGRÁFICAS}

1. Norton, Kevin; Olds, Tim. Antropométrica Ed. Southwood Press, Marrickville. Sidney. 1996.

2. Behnke A.R., Freen B.G., Welham W.C. Specific gravity of healthy men. JAMA, 118: 495-8. 1942.

3. Brozek J. ed. Human body composition. Ann NY Acad Sci, 110: 1-1018. 1963.

4. Siri W.E. Body Composition from fluid space and density. Analysis of Methods. In Techniques of Measuring Body Composition, Washington: Nat Acad Sci. 1961.

5. Matiegka J. The testing of physical efficiency. Am J Phys Anthropol 1921;4:223330.

6. Carter JE. "Somatotype of Olimpic Athletes from 1948 to 1976". Med Sports Sci; 18:80-109. 1984. 
7. Drinkwater, D.T., Martin A.R, Ross W.D., Clarys J.P. Validation by cadaver dissection of Matiegka's equations for the anthropometric estimation of anatomical body composition in human adults. In: Day J.A.P. (ed.) Perspectives in Kinanthropometry, Champaign: Human Kinetics, 1, 221-227, 1984 b. 1984.

8. Drinkwater D.T. An anatomically derived method for he anthropometric estimation of human body composition. PH.D. Thesis Fraser University, 1984 a. 1984.

9. Martin AP, Spenst LF, Drinkwater DT, Clarys JP. Anthropometric estimation of muscle mass in men. Med Sci Sports Exerc 1990;22: 729-33.
10. Doupe MB, Martin AD, Searle MS, Kriellaars DJ, Giesbrecht GG. A new formula for population-based estimation of whole body muscle mass in males. Can J Appl Physiol 1997;22:598-608.

11. Almagia Flores, Atilio Aldo et al . Perfil Antropométrico de Jugadores Profesionales de Voleibol Sudamericano. Int. J. Morphol., Temuco, v. 27, n. 1, marzo 2009.

12. Almagià Flores, Atilio Aldo et al . Perfil Antropométrico de Jugadoras Chilenas de Fútbol Femenino. Int. J. Morphol., Temuco, v. 26, n. 4, dic. 2008.

\begin{abstract}
In 1990, Alan Martin publishes an equation of regresion for the estimate of the muscular mass and the correlation with dissections of 12 corpses, obtaining a determination coefficient (R2) of 0,97 (or, excellent for predecir the mass of the corpses) and the error of standard estimate (EEE) 1,53 kg. In 1997, a Canadian named Malcolm Doupe modified the equation of Martin to calculate the muscular mass of the 23.400 Canadians that were measured in the Canada Fitness Survey of 1981, where did not measure neither the perimeter of medial thigh neither the forearm that the equation of Martin requires, but the perimeters of maximum thigh and arm relaxed. Doupe presents the following equation: muscular Mass $(\mathrm{g})=\operatorname{size}(\mathrm{cm}) *$ $(0,031 *$ MUThG2 $0,064 *$ CCG2 $0,089 *$ CAG2) - 3,006.

The formula for the calculation of the muscular mass of the upper member is M SUP= size $(\mathrm{cm}) *(0,089 * \mathrm{CAG})-3,006$ and for the inferior member and abdomen, M INF= cut $(\mathrm{cm}) *(0,031 *$ MUThG2 $0,064 *$ CCG2 $)-3,006$. Knowing this advantage, apply formula them to two teams of sportsmen, 11 professional players of rugby and 11 professional players of futbol Chilean. We obtain significant differences for $\mathrm{p}>0,05$ between the kilos $(\mathrm{kg})$ of total muscular mass, where, Rugby= $48,1 \mathrm{~kg}$; Football $=42,3 \mathrm{~kg}$ and of the upper member, being Rugby= 16,1 kg; Football= 12,5kg. In the inferior members there are significant differences of $>$ p 0,05 in the percentages (\%) of muscular mass, obtaining Rugby $=35 \%$; Football $=40,9 \%$.

Through this method it is possible to specify not only the total component of muscle mass, but also identify the location greater or lesser presence in upper limbs, Abdomen or inferior member, allowing compare of form more precise the distribution of the muscular mass of different subjects.
\end{abstract}

Key words: Anthropometry, Corporal Composition, Sport.

\author{
Dirigir correspondencia a: \\ Fernando Barraza Gómez \\ Escuela de Educación Física \\ Facultad de Filosofía y Educación \\ Pontificia Universidad Católica de Valparaíso. \\ Chile. \\ fernando.barraza@ucv.cl
}

RECIBIDO 31-10-2009

ACEPTADO 23-12-209 